\title{
3D Printed Millimeter Wave Receiver Integrating a Graphene Subharmonic Mixer and a Diagonal Horn Antenna
}

\author{
Andreea Hadarig, Samuel Ver Hoeye, Carlos Vázquez, René Camblor, Miguel Fernández, \\ George Hotopan, Leticia Alonso and Fernando Las-Heras \\ Área de Teoría de la Señal y Comunicaciones \\ Universidad de Oviedo, Gijón, Spain \\ Email: ahadarig@tsc.uniovi.es
}

\begin{abstract}
This work presents a millimeter/submillimeter wave frequency receiver integrating a graphene subharmonic mixer and a diagonal horn antenna. The device receives the $R F$ signal through the diagonal horn antenna directly connected to the WR-3 input of the mixer. The desired frequency mixing performance is obtained using the non-linear behavior of a fewlayer graphene film placed on a microstrip line gap. Using the internally generated $6^{t h}, 8^{t h}$ and $10^{t h}$ harmonic components of the input signal provided by the WR-28 standard waveguide in the 26-40 GHz the downconversion operation of the RF signal to a $300 \mathrm{MHz}$ intermediate frequency is performed. A prototype of the receiver has been manufactured using high precision 3D printing technology. The performance of this device is characterized taking into account the behavior of the IF power in the 220-330 $\mathrm{GHz}$ band. Measured radiation patterns are also included.
\end{abstract}

\section{INTRODUCTION}

During the last years, millimeter and submillimeter band technologies attracted a special interest among scientists worldwide due to the large number of potential applications supported. Wireless communication, security screening, weapons and drugs detection, cancer exploration are only few of the most exploited fields the waves generated in these bands can be used for.

Traditional semiconductors such as Schottky diodes [1] or Field-Effect Transistors (FETs) [2] are usually used for the generation of millimter/submillimeter wave signals. This work presents a different approach consisting in replacing the traditional solid state devices by graphene layers which provide the same non-linear behavior. Furthermore, in [3] it has been demonstrated that a high order frequency multiplier is able to generate signals in the millimeter/submillimeter wave band in only one stage taking advantage of the non-linearity provided by few-layer graphene. In this paper a subharmonic mixer performing frequency downconversion by mixing an RF signal with a high order harmonic component, concept similar to [4] and [5], of a low frequency local oscillator is implemented based on few layer graphene.

A new approach consisting in the integration of a diagonal horn antenna in the mixer configuration, to minimize power losses, has been used in the present paper. High precision 3D

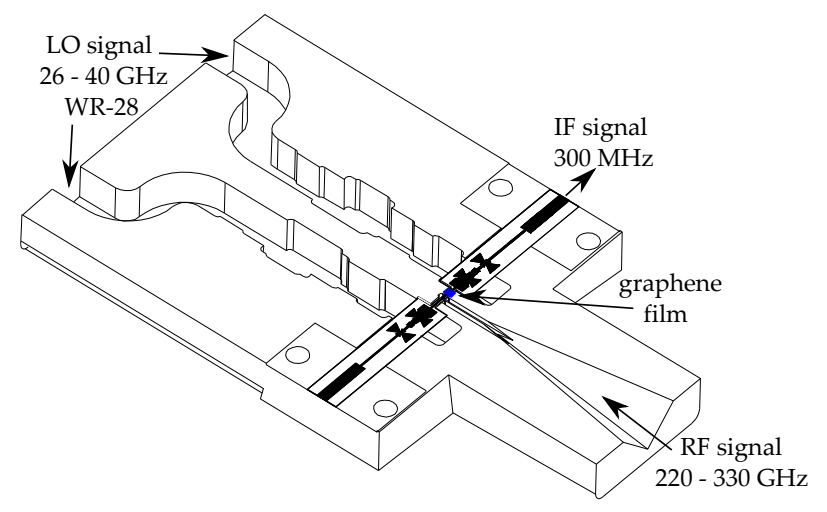

Fig. 1. Receiver topology including the horn antenna for the reception of the RF signal and the subharmonic frequency mixer.

printing technology is utilised in the manufacturing process of the frequency receiver. This new technology reduces the fabrication time and cost as well as enables the mass production.

\section{TOPOLOGY OF THE RECEIVER CONSISTING OF A SubHarmonic MiXer AND A Horn ANTENNA}

The topology of the receiver is presented schematically in Fig. 1. The device receives the input RF signal through a diagonal horn antenna in the frequency band between 220 $330 \mathrm{GHz}$. The input LO signal is received through one of the WR-28 standard waveguides in the frequency band $26-40 \mathrm{GHz}$ while the other one is used to achieve impedance matching in the corresponding band. The $\mathrm{n}^{\text {th }}$ harmonic component $\left(6^{\text {th }}\right.$, $8^{t h}$ and $10^{t h}$ ) of the LO signal is internally generated taking advantage of the graphene behavior. Thus, the frequency downconversion of the RF signal to a $300 \mathrm{MHz}$ IF signal is performed as in (1).

$$
f_{I F}=f_{R F}-n \times f_{L O}
$$

\section{A. Microstrip circuit}

The concept of the frequency mixer is based on the graphene non-linear behavior. For the preparation of the microstrip 


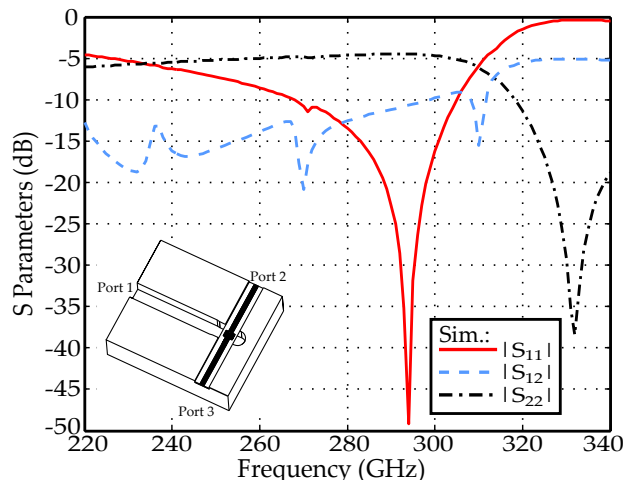

Fig. 2. Simulated scattering parameters of the microstrip to WR-3 waveguide transition.

structure the graphene film was mechanically exfoliated from Highly Oriented Pirolitic Graphite (HOPG) and placed on top of a polyimide substrate with dielectric constant $\varepsilon_{r}=3.5$ and $\tan \delta=0.008$, both measured at $300 \mathrm{GHz}$. Gold contacts are deposited over the graphene layer leaving a gap of $150 \mu \mathrm{m}$ uncovered. A rectangular metallic channel is design to link the three waveguides involved in the topology of the frequency mixer and to host the microstrip structure.

The graphene based frequency mixer has been designed and optimized using the three-dimensional electromagnetic simulator HFSS as it is presented in the following section.

\section{B. Waveguide transitions}

The graphene based subharmonic mixer is simulated using gold as conducting material along the whole path of the microstrip line. Due to the absence of a characterized HFSS model for graphene the simulation of the mixer is done in two different steps.

In the first step a microstrip to WR-3 waveguide transition is designed for the simulation of the RF signal. Good impedance matching is achieved by optimizing the shape of the WR-3 waveguide in the proximity of the microstrip line and the location of the backshort. Moreover, the waveguide modes were avoided in the interior of the rectangular channel by properly selecting its height and width $(0.410 \times 0.120 \mathrm{~mm})$ so as to block the propagation of such modes. The simulated $\mathrm{S}$ parameters of this transition are represented in Fig. 2.

Once optimized, this transition is placed in the middle of the complete circuit design (as presented in Fig. 1). The simulated and measured $\mathrm{S}$ parameters of the reoptimized circuit are presented in Fig. 3. Port 1 and 2 correspond to the standard WR-28 waveguides while port 3 is the IF microstrip port adjacent to port 1 . Note that in the measurement process the graphene device was placed on its corresponding position along the microstrip line.

\section{EXPERIMENTAL RESULTS}

For evaluating the behavior of the graphene based receiver, a prototype of the complete structure including the frequency mixer and the horn antenna has been manufactured and experimentally characterized.

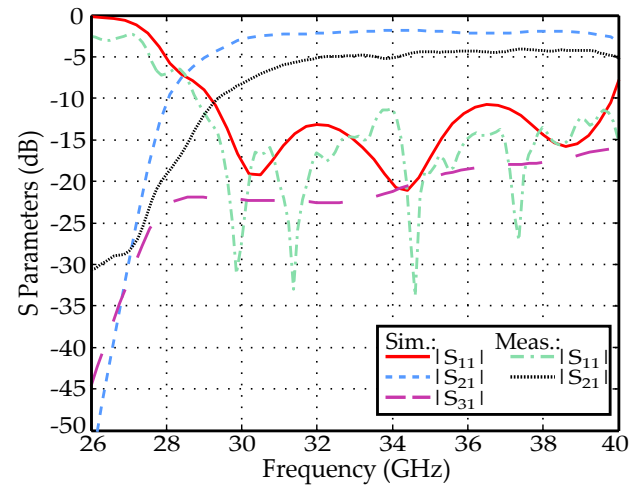

Fig. 3. Simulated and measured scattering parameters of the subharmonic mixer LO signal as presented in Fig. 1.
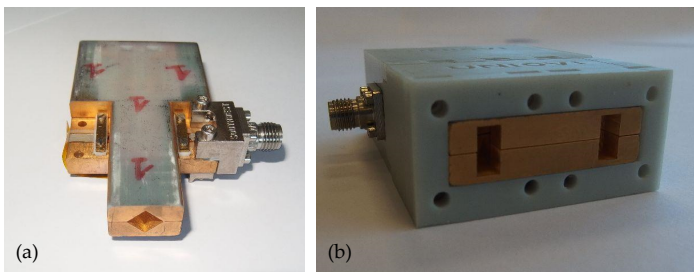

Fig. 4. Manufactured prototype: (a) Input RF without external framing. (b) Input LO with external framing.

The receiver structure was divided into two blocks, resulting from cutting the main structure throughout its transverse plane (one part is presented in Fig. 1). These two blocks were manufactured using a 3D printer with stereolithography technology. The stereolithography technology employs an ultraviolet curable photopolymer called resin and an ultraviolet projector to build parts layer by layer. When the building process is complete, the $3 \mathrm{D}$ object obtained is immersed in a chemical bath in order to eliminate the resin excess, and cured in an ultraviolet oven.

This technology is less expensive and decreases significantly the fabrication time of the prototypes if compared with the other technologies used in literature [3]-[5]. Moreover, repetitive prototypes with the same fabrication precision can be manufactured at a low cost.

The necessary conductivity along the whole structure of the receiver was provided through the deposition of a thin layer of gold particles using a sputtering process.

The microstrip line integrating the non-linear component is prepared and mounted in the interior of the rectangular channel where is carefully aligned by means of a high precision pick and place system. After this process, the two blocks are assembled together and introduced in an external framing designed in such way that at the WR-28 input waveguide it gives the shape of its corresponding standard flange (type UG387/UM). Images of the prototype are presented in Fig. 4.

\section{A. Measurement setup}

The mixing operation of the receiver has been evaluated using the measurement setup presented in Fig. 5. The input LO 


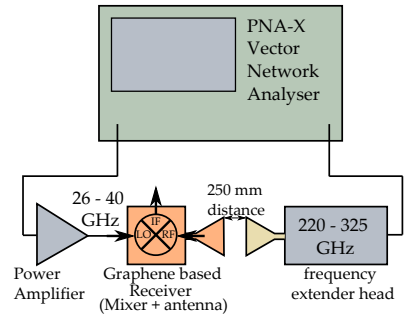

Fig. 5. Schematic diagram of the measurement setup.

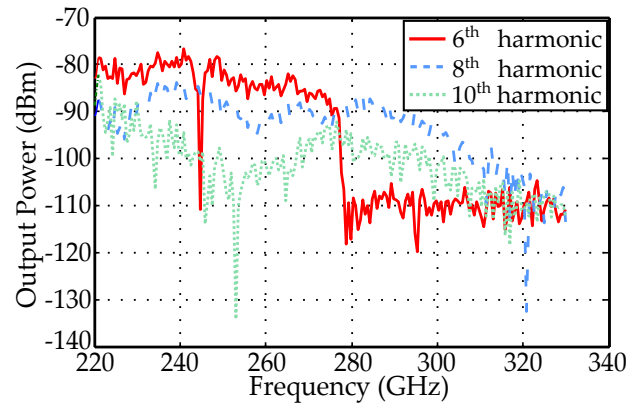

Fig. 6. IF output power of the subharmonic mixer, measured for different harmonic orders of the LO signal.

signal of the receiver in the $\mathrm{K}_{a}$ band is generated by the vector network analyser and amplified up to the power level $\mathrm{P}_{i n}=20$ $\mathrm{dBm}$. Furthermore, the RF input signal is received through the diagonal horn antenna integrated in the configuration of the receiver. This signal is generated by the frequency extender head connected to the PNA-X and transmitted to the receiver using an identical horn antenna placed in the far field region, at a distance of $250 \mathrm{~mm}$. The power level of the RF signal varies between -21 and $-12 \mathrm{dBm}$.

The output signal of the receiver is measured at an intermediate frequency $\mathrm{f}_{I F}=300 \mathrm{MHz}$. The frequency downconversion operation is performed by mixing the RF signal with the $6^{\text {th }}, 8^{t h}$ and $10^{\text {th }}$ harmonic components of the LO signal.

\section{B. Receiver performance}

The IF output power of the receiver is measured in downconversion of an WR-3 input RF signal using a high order harmonic component of an WR-28 input signal used as local oscillator. The measured results are presented in Fig. 6.

Considering the impedance matching of the WR-28 input which is limited to $29-40 \mathrm{GHz}$ (Fig. 3) the RF signal required for the mixer downconversion when using different high order harmonic components of the LO signal takes different frequency ranges within the WR-3 frequency band. The RF frequency ranges calculated using (1) are: $220-240.3 \mathrm{GHz}$ for the mixer downconversion using the $6^{\text {th }}$ harmonic component of the LO; $232.3-319.7 \mathrm{GHz}$ for $8^{\text {th }}$ harmonic component and $290.3-330 \mathrm{GHz}$ for $10^{\text {th }}$ harmonic component.

\section{Radiation patterns}

Fig. 7 presents the $\mathrm{E}$ and $\mathrm{H}$ plane radiation patterns of the receiver, measured at $270 \mathrm{GHz}$. Good agreement between the

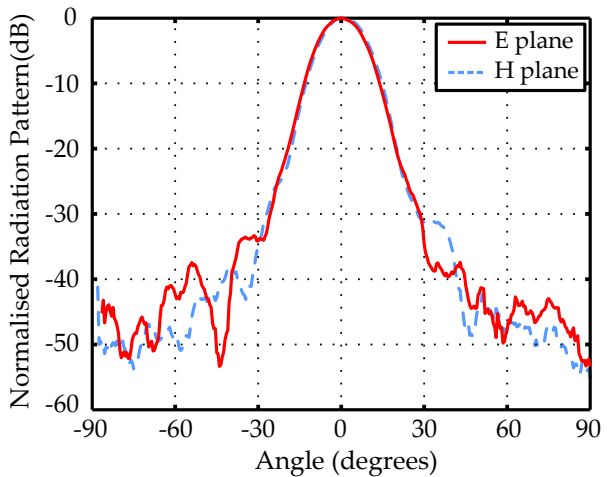

Fig. 7. Radiation patterns measured at $270 \mathrm{GHz}$ (E and $\mathrm{H}$ plane).

radiation characteristics of $\mathrm{E}$ and $\mathrm{H}$ plane is obtained.

\section{CONCLUSION}

The prototype of a millimeter/submillimeter wave receiver based on a graphene subharmonic mixer and a diagonal horn antenna has been presented. The mixer downconverts an input RF signal in the WR-3 frequency band (220 - $330 \mathrm{GHz})$ to an intermediate frequency (300 MHz) using a high order harmonic component ( $n=6,8$ and 10) of an input LO signal in the WR-28 band (26.5 - $40 \mathrm{GHz})$. High precision 3D printing techniques have been used to minimize the fabrication cost and time of the prototype. The mixer receives its RF signal through a horn antenna placed in the far field region, at a distance of $250 \mathrm{~mm}$ from the transmitter. Radiation patterns of the receiver are measured and presented together with the IF output power internally generated. The maximum power level achieved with this prototype is around $-75 \mathrm{dBm}$.

\section{ACKNOWLEDGMENT}

This work has been supported by the European Union Seventh Framework Programme (FP/2007-2013) under grant agreement number 600849 , by the "Ministerio de Ciencia e Innovación" of Spain/FEDER under projects TEC2008-01638/TEC, TEC2011/24492, IPT-2011-0951390000 and CONSOLIDER-INGENIO CSD2008-00068, grant AP2012-2020, by the Gobierno del Principado de Asturias (PCTI)/FEDER-FSE under projects EQUIP08-06, FC09-COF09-12, EQUIP10-31, GRUPIN14-114, grant BP13034 and by Cátedra Telefónica - Universidad de Oviedo.

\section{REFERENCES}

[1] C. Lee, et al., "A Wafer-Level Diamond Bonding Process to Improve Power Handling Capability of Submillimeter-Wave Schottky Diode Frequency Multiplier," IEEE MTT-S International Microwave Symposium Digest, 7-12 June 2009, pp 957-960, Boston, MA.

[2] E. Camargo, "Microwave and Millimeter-wave FET Frequency Mulipliers and Harmonic Oscillators," Artech House, UK, 1998.

[3] A. Hadarig, et al., "Experimental analysis of the high order harmonic components generation in few-layer graphene," App. Phy. A, Vol. 118, No. 1, pp 83-89, 2015.

[4] C. Vazquez, et al., "High-Order Subharmonic Millimeter-Wave Mixer Based on Few-Layer Graphene," Microwave Theory and Techniques, IEEE Transaction on, Vol. 63, No. 4, pp 1361-1369, April 2015.

[5] C. Vazquez, et al., "Millimeter Wave Subharmonic Mixer Based on Graphene," 2014 ITS, 17-20 Aug., pp 1-5, 2014. 